\title{
Einführung eines Qualitätsmanagement-Systems in einem molekularbiologischen Labor einer Hochschule
}

\author{
Stefan Weinert, Ingolf Wohlfahrt, Andrea Schmid und Marcus Frohme
}

\section{Zusammenfassung}

Zertifizierte Qualitätsmanagementsysteme (QM-Systeme) sind in akademischen Forschungslaboren bisher kaum vertreten. Hier ist vor allem die Bedeutung eines zertifizierten Labors vor dem Hintergrund der Veröffentlichung wissenschaftlicher Publikationen scheinbar bedeutungslos.

Im industriellen Bereich hingegen sind QM-Systeme weitaus häufiger eingeführt. Labore im akademischen Bereich stehen jedoch grundsätzlich in der Verantwortung, schonend mit den Ressourcen des Staates umzugehen, und haben darüber hinaus das Bestreben, Forschungs-/Industrieaufträge einzuwerben.

Mit dieser Publikation wird gezeigt, wie durch die Einrichtung eines QM-Systems und der Einhaltung der normativen Vorgabe ein verbesserter schonender Umgang mit staatlichen Ressourcen möglich ist, die Ergebnissicherheit erhöht, Betriebsabläufe optimiert, die Chancen für Studenten auf dem Arbeitsmarkt verbessert werden und mehr Forschungs-/Industrieaufträge akquiriert werden können. Es wird dargestellt, wie ein QM-System in einem Labor eingerichtet werden kann. Dabei wird erläutert, welche QM-Regelwerke für ein Labor im Hochschulbereich in Betracht kommen. Zudem wird eine Methodik in Form eines Projektplans vorgestellt, mit dem die Anforderungen der Norm strukturiert umgesetzt werden können.

Das QM-System wurde im Labor für Molekularbiologie und Funktionelle Genomik an der Technischen Hochschule Wildau [FH] eingeführt und im Rahmen der Zertifizierung der Hochschule in das Audit einbezogen.

\section{Abstract}

Up to now certified Quality-Management-Systems (QM-system) are hardly prevalent in university research laboratories. Even if a laboratory is certified, it seems to have no meaning for a scientific publication originating from this laboratory.

In the industrial sector QM-systems are a lot more common. Basically, academic laboratories have to use their rescources, provided primarily by govermental funding, very sparingly and responsible. Furthermore universities have a strong endeavour to accquire funding for further research.

We demonstrate here, how the establishment of a QMsystem, and compliance to normative requirements, can improve the economic use of governmental funding. Furthermore a QM-system can ensure result reliability and work routines can be optimized. In addition the job perspectives for graduates as well as the possibilities to aquire funding will be improved. We present how a QM-system can be established in a lifescience-laboratory. Thereby it will be outlined which regulations are applicable to an academic research lab. Following that, a method to implement a QM-system will be presented. This method is shown as a project schedule and explains the requirements that are necessary to establish quality standards. The QM-system was implemented in the Laboratory for Molecular Biology and Functional Genomics of the Technical University of Applied Sciences Wildau and was included within the scope of the certification of the university in the audit.

\section{Einleitung}

\subsection{Situationsbeschreibung}

Qualitätsmanagement (QM) als Tätigkeit leitet und lenkt eine Organisation mit dem Ziel, die Anforderungen der interessierten Parteien zu erfüllen (DIN EN ISO 9000:2005). Hierzu ist die Einführung eines QM-Systems notwendig.
In den Life Sciences spielen QM-Systeme eine große Rolle (Schweitzer 2009). Der Gesetzgeber schreibt z. B. hohe Qualitätsstandards bei der Entwicklung, Erprobung und Herstellung von Arzneimitteln vor. Durch die eingeführten QM-Systeme soll garantiert werden, dass die Standards eingehalten werden. Bewiesen wird die Einhaltung der hohen Qualitätsstandards durch eine Zertifizierung oder Akkreditierung (Cassel 2000: 6/1). 
Eine erfolgreiche Zertifizierung ist die formelle Bestätigung, dass festgelegte Anforderungen an z. B. ein Produkt oder Managementsystem erfüllt sind. Durch eine Akkreditierung hingegen besitzt die Organisation die formale Kompetenz, für ausgewiesene Leistungen Bewertungen durchzuführen. Für Labore basiert die Akkreditierung auf der fachlichen Kompetenz, technischen Ausstattung und der Konformität zur Norm (GLP-INFO 11, 2004).

Im universitären Forschungsbereich sind akkreditierte bzw. zertifizierte QM-Systeme bisher kaum eingeführt. Die vorrangigen Ziele von universitären Forschungslaboren bestehen darin, durch Forschungsaufträge des Staates und von Kooperationspartnern neue wissenschaftliche Erkenntnisse zu erlangen, Studenten auszubilden und ggf. vorhandene Analysemethoden am Markt anzubieten. Um einen Forschungsauftrag zu erlangen, wird zunächst ein entsprechender Antrag gestellt, welcher sich dann gegenüber anderen Mitbewerbern behaupten muss. Mit einer positiven Entscheidung kann das Labor Forschungsgelder in Höhe von mehreren hunderttausend Euro erhalten. In diesem Rahmen wird erwartet, dass mit den übergebenen Ressourcen vertrauensvoll umgegangen wird und erbrachte Erkenntnisse möglichst sicher bzw. vertrauenswürdig sind. Mit den gewonnenen Erkenntnissen aus der Forschungstätigkeit gehen dann neue Publikationen einher, welche so zum wissenschaftlichen Fortschritt beitragen. Für die Veröffentlichung von wissenschaftlichen Arbeiten erscheint ein QM-System bisher nicht notwendig. Hier werden die Glaubhaftigkeit durch das Lektorat, das Prestige der Arbeitsgruppe und die Angabe von Material und Methoden gegeben.

Durch das eingeführte QM-System in einem universitären Labor soll nicht die Glaubhaftigkeit der Publikationen erhöht werden. Vielmehr wird hier die Verbesserung der organisatorischen Strukturen angestrebt, um so das Vertrauen in die durchgeführten Tätigkeiten gegenüber dem Staat, Kooperationspartnern und den eigenen Mitarbeitern zu erhöhen.

Das QM-System wurde im Labor für Molekularbiologie und Funktionelle Genomik der Technischen Hochschule Wildau [FH] eingeführt. Im Rahmen der erstmaligen Zertifizierung des QM-Systems der Hochschule nach ISO 9001 wurde das QM-System des Labors in Bezug auf die Prüfmittellenkung in die Auditierung der Hochschule einbezogen. Die Hochschule sowie die Regelungen zur Prüfmittellenkung des Labors wurden im Juni durch die »Deutsche Gesellschaft zur Zertifi- zierung von Managementsystemen « (DQS) erfolgreich zertifiziert. Damit hat der Aufbau eines spezifischen QM-Systems im Labor für Molekularbiologie und Funktionelle Genomik Pilotcharakter für die weiteren Labore der Hochschule.

\subsection{Problemdarstellung}

In den Life Sciences spielen mehrere QM-Systeme eine Rolle, welche je nach Anwendung auch gesetzlich gefordert sind. Durch die unterschiedlichen Anwendungsbereiche und Inhalte des QM-Systems werden die Anforderungen der interessierten Parteien (z. B. Industriepartner) unterschiedlich stark erfüllt. Daher muss zunächst das geeignetste QM-System für das Labor identifiziert werden.

Um einen normenkonformen Zustand herstellen zu können, müssen die Anforderungen der normativen Vorgaben (z. B. ISO 17025 oder Unfallverhütungsvorschriften) identifiziert und mit den bestehenden Regelungen abgeglichen werden. Da die Dauer zur Einführung eines QM-Systems ca. 8 - 24 Monate beträgt, wird eine Methode dargestellt, mit der eine kontinuierliche, strukturierte und zielgerichtete Einführung gestaltet werden kann (Wohlfahrt 2008: 164).

\subsection{Interner Anspruch und Ziel}

Das molekularbiologische Labor der TH Wildau führt u. a. Genexpressionsanalytik in Kooperation mit akademischen und industriellen Partnern durch. Um der wachsenden Nachfrage des Marktes gerecht zu werden, sollen ein QM-System eingeführt und die Nachweisführung zur Ergebnissicherheit durch eine konforme Laborführung gewährleistet werden. Hierdurch wird angestrebt, die Lage zur Akquirierung neuer Kooperationen zu verbessern und Betriebsabläufe zu optimieren. Außerdem wird erwartet, dass mit dem eingeführten QM-System ein vertrauensvollerer Umgang mit den zur Verfügung stehenden Ressourcen gewährleistet wird. Des Weiteren soll die studentische Ausbildung von einem gelebten QM-System profitieren.

\section{Auswahl des QM-Systems}

Vor der Einführung eines QM-Systems steht zunächst die Auswahl des QM-Regelwerkes. In den Life Sciences existieren mehrere QM-Regelwerke mit unterschiedlichen Anwendungsbereichen (z. B. Arzneimittelherstellung oder medizinische Diagnostik). Für universitäre 


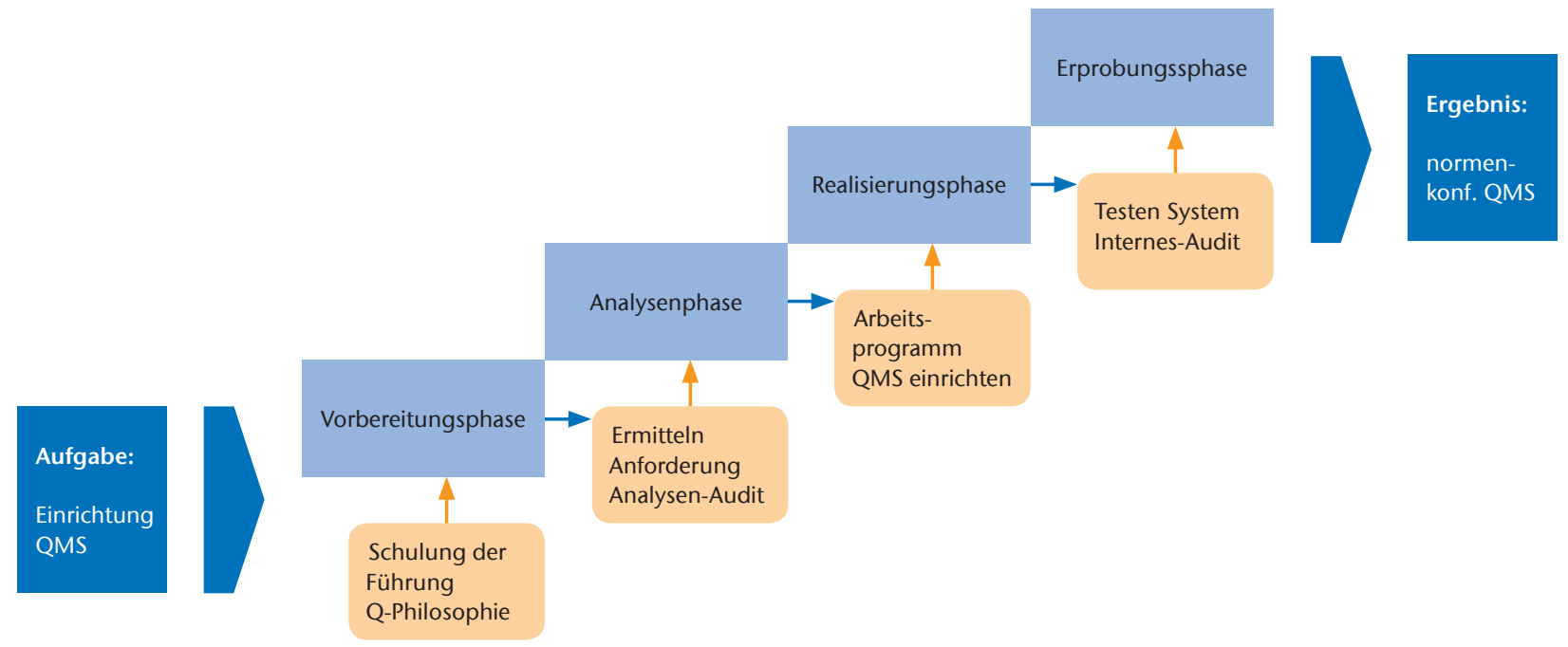

Abb. 1: Phasenmodell zur Einführung eines QM-Systems (Wohlfahrt 2008: 164)

Forschungslabore sind prinzipiell die DIN EN ISO 9001 (ISO 9001) oder DIN EN ISO/IEC 17025 (ISO 17025) geeignet.

Die ISO 9001 kann für alle Organisationen verwendet werden. Sie findet dort Anwendung, wo ein Nachweis der Qualitätsfähigkeit erbracht werden soll, und dient dem Zweck, Vertrauen zu schaffen. Im Mittelpunkt der Norm stehen der Kunde, die Erfüllung der zutreffenden gesetzlichen Anforderungen und die kontinuierliche Verbesserung der Organisation (Sagert 2003: 12).

Die ISO 17025 enthält alle Anforderungen an Prüf-/ Kalibrierlaboratorien einschließlich der Probenentnahme. Sie dient dazu, die Ergebnissicherheit durch die Kompetenz des Personals, der Ausrüstung und der anzuwendenden Verfahren zu beweisen (DIN EN ISO/IEC 17025:2005). Damit gewährleistet sie die Genauigkeit und Vergleichbarkeit von Prüfergebnissen und bezieht sich auf normative Verfahren sowie auf Verfahren, die in den Laboratorien entwickelt wurden. Von 2000 bis 2009 betrug der durchschnittliche Anstieg der Akkreditierungen durch die Deutsche Akkreditierungsstelle Chemie 23,6\% (DACH-N, 2008).

Da für das molekularbiologische Labor die Ergebnissicherheit der erbrachten Genexpressionsanalytik für die Kooperationspartner im Vordergrund steht, wurde die ISO 17025 als einzuführendes QM-System ausgewählt.

Bei der weiteren Einführung des QM-Systems sind zudem die rechtlichen Vorgaben (z. B. Unfallverhütungsvorschriften) zu beachten und einzubeziehen. So fordert der Gesetzgeber z. B., dass Zentrifugen ab einer bestimmten Leistung jährlich durch zertifizierte Techniker gewartet werden.

\section{Einführung eines QM-Systems}

Ausgangspunkt für die Einführung eines QM-Systems ist die positive Entscheidung des Laborleiters. Die Einführung ist ein komplexes Projekt und muss entsprechend gut geplant werden (Raguse 2001). Das Projekt wurde in vier Phasen gegliedert: Vorbereitungsphase, Analysenphase, Realisierungsphase und Erprobungsphase (Wohlfahrt 2008: 164). Abbildung 1 zeigt eine Übersicht zum Inhalt der einzelnen Phasen.

\subsection{Vorbereitungsphase}

Zunächst wird die Situation (z. B. Marktbetrachtung) zur Einführung des QM-Systems ermittelt und der Laborleitung die Vor- und Nachteile des QM-Systems dargestellt. Des Weiteren werden in dieser Phase die Unternehmensphilosophie (Q-Politik) erarbeitet und die Einführung des QM-Systems als Projekt zeitlich definiert. Die Ausarbeitung der Q-Politik stellt den zentralen Punkt bei der Einführung eines QM-Systems dar. Aus den gebildeten Qualitätsgrundsätzen der Q-Politik leitet sich der weitere Handlungsbedarf ab. Ein Qualitätsgrundsatz kann z. B. sein, dass das Labor für die Fehlerfreiheit seiner Analysen steht und damit wirbt. Hieraus leitet sich u. a. die Notwendigkeit ab, Maßnahmen zur Prävention zu treffen, Mitarbeiter besonders zu schulen und entstandene Fehler zu lenken. Die Erfüllung der Anforderung aus der Norm, wie z. B. ein Verfahren für vorbeugende Maßnahmen für den Schulungsbedarf und die Lenkung von Fehlern einzuführen, wird für die Mitarbeiter verständlicher. Das Aufstellen der Q-Politik gibt auf diese Weise die Richtung zur Einführung des QM-Systems vor. Um die Q-Politik aufstel- 
len zu können, müssen die interessierten Parteien des Unternehmens identifiziert und deren Anforderungen dargstellt werden. Als interessierte Parteien des molekularbiologischen Labors wurden Industrieunternehmen, Institute, Mitarbeiter, Lieferanten, die Gesellschaft und der Staat identifiziert.

\subsection{Analysephase}

In dieser Phase werden zunächst die SOLL-Anforderungen aus der Norm und die rechtlichen Anforderungen gesammelt. Anschließend wird in einem Analyse-Audit der IST-Bestand aufgenommen und mit den SOLL-Anforderungen abgeglichen. Im molekularbiologischen Labor wurden die SOLL-Anforderungen hierzu in Schwerpunkte (z. B. Beschaffung) gegliedert. Anschließend erfolgte der Abgleich mit den vorhandenen Regelungen durch die betreffenden Mitarbeiter.

\subsection{Realisierungsphase}

Inhalt der Realisierungsphase ist das Aufstellen des Arbeitsprogramms. Das Arbeitsprogramm zeigt die Abarbeitungsreihenfolge zur Einführung des QM-Systems. Es empfiehlt sich, das Arbeitsprogramm entsprechend der eigenen Vorstellung und Kapazitäten in Prioritäten zu unterteilen (Brunner und Wagner 2008: 65). Hierzu wurde für das molekularbiologische Labor eine Methode entwickelt, um die Prioritäten in Zahlen zu fassen.

\section{Methode zur Ermittlung der Prioritäten}

Die Ermittlung der Prioritäten wurde angelehnt an die Qualitätsmanagementmethode »Fehler-Möglichkeit und Einfluss-Analyse . In der angewandten Methode wurden die vier Kategorien Fehlerpotenzial (bezogen auf die Prüfung), Förderung der Akzeptanz für das QMSystem bei den Mitarbeitern, bereits bestehende Vorlagen (z. B. Muster Verfahrensanweisungen an der TH Wildau) und allgemein empfundene Dringlichkeit beurteilt. Hierbei wurden für jede Kategorie ein bis zehn Punkte vergeben. Im Anschluss wurden alle vier Werte multipliziert. Je höher das Ergebnis war, desto höher wurde die Priorität eingestuft.

\section{Einrichten des QM-Systems}

Über das Arbeitsprogramm erfolgt anschließend das Einrichten und Implementieren des QM-Systems. Hier werden notwendige Dokumente erstellt, Regelungen getroffen und die Mitarbeiter entsprechend geschult. Im molekularbiologischen Labor wurde hierbei zunächst eine festgelegte Reihenfolge (Organisations- struktur, QM-Handbuch und Lenkung der Dokumente/ Aufzeichnungen) eingehalten. Die weitere Einführung orientierte sich an den ermittelten Prioritäten des Arbeitsprogramms.

\section{Festgelegte Reihenfolge}

Zunächst wurde die Organisationsstruktur des Labors festgelegt. Hier werden die Hierarchie der Organisation dargestellt und die Verantwortungsbereiche und Befugnisse der Mitarbeiter ausgearbeitet. Dies ist die Vorraussetzung, um Aufgaben innerhalb der zu regelnden Prozesse zuzuweisen. Nachdem die Organisationsstruktur festgelegt worden war, wurde begonnen das QMHandbuch zu erstellen. Es beinhaltet die Q-Politik und beschreibt das komplette QM-System einer Organisation (Kamiske und Brauer 2008: 218). Bei der Einführung des QM-Systems werden alle bearbeiteten Schwerpunkte aus dem Arbeitsprogramm nach deren Erfüllung, sukzessiv in das QM-Handbuch aufgenommen. Anschließend wurde die Lenkung von Dokumenten und Aufzeichnungen geregelt. So wird gewährleistet, dass alle Dokumente und Aufzeichnungen, die zur Einführung benötigt werden, nach demselben Schema und derselben Form erstellt werden und somit konform zur Norm sind. Der festgelegte Ablauf ist nach der Regelung der Lenkung von Dokumenten und Aufzeichnungen abgeschlossen.

\section{Beispiel zur Umsetzung eines Schwerpunkts aus der Norm}

Um die Umsetzung der einzelnen Anforderungen aus der Norm beispielhaft näher zu bringen, wird im Folgenden auf die Prüfmittellenkung eingegangen. Ziel der Prüfmittellenkung ist es, den Einsatz der Prüfmittel und deren Tauglichkeit permanent sicherzustellen.

Die ISO 17025 fordert, dass die Prüfmittel in der Lage sind, die Prüfmerkmale mit der erforderlichen Genauigkeit zu bestimmen (DIN EN ISO/IEC 17025:2005). Die Aufnahme der Spezifikationen (Messunsicherheit/bereich) erfolgte in Zusammenarbeit mit der verantwortlichen Mitarbeiterin. Hierzu wurden der Ablauf der Analyse in einem Flussdiagramm verdeutlicht und alle zu bestimmenden Prüfmerkmale in den einzelnen Analysenschritten (z. B. Vorbereitung oder Isolierung) eruiert.

Haben Prüfmittel einen signifikanten Einfluss auf die Messgrößen und das Ergebnis, müssen diese überwacht werden. Hierzu wird von der Norm verlangt, dass ein Kalibrier-/Wartungsprogramm eingeführt wird 
(DIN EN ISO/IEC 17025:2005). Bei der Ermittlung, wie das Prüfmittel zu überwachen ist, wurden im molekularbiologischen Labor drei Arten unterschieden: die periodische Eigenüberwachung/Fremdüberwachung und die prozessbegleitende Kontrolle (QMV 2008). Die prozessbegleitende Kontrolle eines Prüfmittels ist vor jedem Gebrauch notwendig. Hier wird das Prüfmittel z. B. durch Reinigen oder Vorwärmen in den betriebsbereiten Zustand versetzt. Wohingegen eine periodische Überwachung nur in festgelegten Abständen notwendig ist.

Ist eine anstehende Überwachung vor Inbetriebnahme nicht erfolgreich oder das Prüfmittel liefert unglaubwürdige Ergebnisse, ist es zu sperren und es eindeutig als gesperrtes Prüfmittel zu kennzeichnen (DIN EN ISO/IEC 17025:2005). In diesem Zusammenhang ist ein Sperraufkleber entworfen worden, welcher ggf. am Prüfmittel angebracht wird.

\section{Projektplan zur weiteren Einführung des QM-Systems} Um das Einführen des QM-Systems zu erleichtern, sollten die zu bearbeitenden Schwerpunkte als Teilprojekt betrachtet werden. Hierzu wurde ein entsprechender Projektplan entwickelt. Der Projektplan orientiert sich am Vorgehen zur Einführung eines QM-Systems (Wohlfahrt 2008: 164). Er präzisiert das Vorgehen zur Umsetzung der einzelnen Schwerpunkte des Arbeitsprogramms.

Beim Vorgehen zur Einführung sollten durch den Prozessverantwortlichen zunächst noch einmal die jeweiligen Anforderungen aus der Norm veranschaulicht und die bisherigen Regelungen und Dokumente gesammelt werden. Anschließend sollte der betrach- tete Prozess vorzugsweise visuell dargestellt werden. Wenn der Ablauf des Prozesses verdeutlicht ist, können notwendige fehlende Standard Operating Procedures (SOP) bzw. Aufzeichnungen ermittelt und erstellt werden. Nach der Ausarbeitung der Dokumente und Regelungen sollten die bestehenden Anforderungen der Norm mit dem Lösungskonzept abgeglichen werden. Wurden Anforderungen nicht berücksichtigt, ist eine Anpassung durchzuführen. Sind die fehlenden Anforderungen aufgenommen worden und es bestehen noch weitere Unklarheiten die Interpretation betreffend, ist es vorteilhaft, einen externen Berater einzubeziehen. Es ist zu empfehlen, mehr Arbeit in die Vorbereitung und den Abgleich zu investieren, um später aufwendige Korrekturen am Lösungskonzept zu vermeiden.

Wurden die Anforderungen umgesetzt, kann das Lösungskonzept in der Praxis an einigen Beispielen getestet werden. Auftretende Beeinträchtigungen werden dabei sofort aufgenommen. Ist der Test beendet, sind die erforderlichen Verbesserungen und notwendige Veränderungen einzuarbeiten. Anschließend sollte das Lösungskonzept noch einmal mit den Anforderungen der Norm abgeglichen werden.

Wurde das Lösungskonzept abschließend überarbeitet, wird der entsprechende Abschnitt im QMHandbuch erstellt. Nach der Erstellung des QM-Handbuch-Abschnitts können das Lösungskonzept bzw. die Dokumente freigegeben werden. Der Projektplan in Abbildung 2 verdeutlicht dieses Vorgehen noch einmal.

Wurden alle Forderungen an das einzuführende QM-System umgesetzt und freigegeben, ist die Realisierungsphase abgeschlossen.

\begin{tabular}{|c|c|c|c|c|c|c|c|c|c|c|c|c|c|c|c|c|c|c|c|c|c|c|}
\hline \multirow[t]{2}{*}{ Nr. } & \multirow{2}{*}{\multicolumn{2}{|c|}{ Vorgangs name }} & \multicolumn{7}{|c|}{ 06. Jul'09 } & \multicolumn{7}{|c|}{ 13. Jul'09 } & \multicolumn{6}{|c|}{ 20. Jul'08 } \\
\hline & & & $s$ & $M$ & $D$ & $M$ & D & $s$ & $s$ & $M$ & $D$ & $M$ & $D$ & $F$ & $s$ & $s$ & $M$ & $D$ & $M$ & $D$ & $\mathrm{~F}$ & $s$ \\
\hline 1 & \multicolumn{2}{|l|}{ Anforderung ermitteln } & & & & & & & & & & & & & & & & & & & & \\
\hline 2 & \multicolumn{2}{|c|}{ Sammeln bisheriger D okumente/R eglungen } & & & & & & & & & & & & & & & & & & & & \\
\hline 3 & \multicolumn{2}{|c|}{ Prozess darstellen (Schnittstellen, Personen, Ress ourcen) } & & & & & & & & & & & & & & & & & & & & \\
\hline 4 & \multicolumn{2}{|c|}{ U msetzung d. Anforderungen (Erarbeiten d. Dokum ente) } & & & & & & & & & & & & & & & & & & & & \\
\hline 5 & \multicolumn{2}{|c|}{ Meilenste in Abgleich mit Anforderungen } & & & & & & $\checkmark 10$ & & & & & & & & & & & & & & \\
\hline 8 & \multicolumn{2}{|c|}{ ggf. Konsultation $m$ it Berater } & & & & & & & & & & & & & & & & & & & & \\
\hline 7 & \multicolumn{2}{|c|}{ Testen der Umsetzung mit Beispiel } & & & & & & & & & & & & & & & & & & & & \\
\hline 8 & \multicolumn{2}{|l|}{ Bewertung d. Umsetzung } & & & & & & & & & & & & & & & & & & & & \\
\hline 9 & \multicolumn{2}{|c|}{ Abgleich Erfüllung d. Anforderungen } & & & & & & & & & & & & & & & & & & & & \\
\hline 10 & \multicolumn{2}{|l|}{ Ü berarbeiten d. Umsetzung } & & & & & & & & & & & & & & & & & & & & \\
\hline 11 & \multicolumn{2}{|c|}{ Erstellen d. QMH Abschnittes } & & & & & & & & & & & & & & & & & & & & \\
\hline 12 & \multicolumn{2}{|l|}{ Freigabe } & & & & & & & & & & & & & & & & & & & 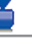 & \\
\hline \multirow{3}{*}{\multicolumn{2}{|c|}{$\begin{array}{l}\text { Projekt: Einführung_Abschnitte } \\
\text { Datum: Mo 06.07.09 }\end{array}$}} & Vorgang & & & & \multicolumn{3}{|c|}{ Meilenste in } & $<$ & & & & \multicolumn{4}{|c|}{ Externe Vorgänge } & E & & & & & \\
\hline & & Unterbrechung & 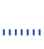 & " & 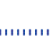 & \multicolumn{3}{|c|}{ Sammelvorgang } & & & & & \multicolumn{4}{|c|}{ Externer Meilenstein } & $\theta$ & & & & & \\
\hline & & In Arbeit & & & & \multicolumn{4}{|c|}{ Projekts am metvorgang } & & & $\nabla$ & \multicolumn{4}{|c|}{ Stichtag } & 凡 & & & & & \\
\hline
\end{tabular}

Abb. 2: Projektplan zur Umsetzung der Schwerpunkte des Arbeitsprogramms 


\subsection{Erprobungsphase}

Mit der Erprobungsphase erfolgt die Umsetzung der eingerichteten Prozesse aus der Realisierungsphase. Durch ein internes Audit werden die Umsetzungen zwei bis drei Monate nach Umsetzungsbeginn auf ihre Wirksamkeit untersucht. Notwendig werdende Veränderungen werden dann erlassen und realisiert. Dadurch soll ein normenkonformer Zustand der Organisation erreicht werden.

Nach der Erprobungsphase erfolgt das externe Audit durch die Zertifizierungs-/Akkreditierungsstelle. Wenn diese die Konformität zur Norm feststellt, wird das Zertifikat ausgestellt. Die Kosten sind in der Regel aufwandsabhängig und betragen für eine Zertifizierung ca. 1800 Euro und für eine Akkreditierung ca. 2600 Euro pro Jahr (Malow 2009; Wisser 2009).

\section{Diskussion}

\subsection{Auswahl des QM-Systems}

Das molekularbiologische Labor der TH Wildau [FH] strebt an, die Betriebsabläufe zu verbessern und darüber hinaus die Ergebnissicherheit für seine Kooperationspartner durch eine normenkonforme Laborführung zu bestätigen. Zur Einführung eines QM-Systems für universitäre Forschungslabore kommen die ISO 9001 und die ISO 17025 in Betracht. Universitäre Forschungslabore, welche neue Methoden entwickeln bzw. verbessern, sollten eine Zertifizierung nach ISO 9001 anstreben. Handelt es sich bei der angewandten Methode um eine Routineanwendung mit dem Ziel, bestmögliches Vertrauen in das Ergebnis zu erhalten, ist eine Akkreditierung nach ISO 17025 nahe zu legen. Daher wurde die ISO 17025 als einzuführendes QM-System im molekularbiologischen Labor ausgewählt.

\subsection{Einführung des QM-Systems}

\section{Phasen zur Einführung eines QM-Systems und Q-Politik}

Bei der Einführung eines QM-Systems ist die Gliederung in die vier Phasen (Vorbereitung, Analysen, Realisierung und Erprobung) zielführend und zu empfehlen. Es ist wichtig, dass die Einführung zeitlich definiert und strukturiert ist, um eine klare Zielvorgabe zu schaffen. Der Laborleiter verleiht auf diese Weise von Anfang an mit Nachdruck die Bedeutung für alle Beteiligten zur Einführung des QM-Systems. Das Aufstellen der Q-Politik sollte bei der Einführung besonderen Wert einnehmen. Mit der Einführung der Q-Politik werden, gespiegelt an den Anforderungen der interessierten Parteien, die Aufgaben und die Mission des Unternehmens deutlich. Wenn vor allem der Laborleiter das Verständnis zur Erfüllung dieser Anforderungen entwickelt hat, ist die Einhaltung der Norm fast obligatorisch. Gerade die ISO 17025 stellt von diesem Standpunkt aus einen Leitfaden für Labore zur Erbringung ihrer Leistung dar.

\section{Einrichtung des QM-Systems}

Bei der folgenden Einrichtung des QM-Systems in der Realisierungsphase hat es sich bewährt, eine festgelegte Reihenfolge (Organisationsstruktur, QM-Handbuch und Lenkung der Dokumente/Aufzeichnungen) einzuhalten. Vor allem das Schaffen klarer Organisationsstrukturen und die Vorgabe zur Dokumentation des QM-Systems stellen die Vorraussetzung zur weiteren Einführung dar. Wenn diese Voraussetzungen erbracht sind, können die weiteren Anforderungen aus der Norm, wie z. B. Regelungen zur Beschaffung oder Prüfmittellenkung, umgesetzt werden.

Durch den vorgestellten Projektplan zur Umsetzung der Schwerpunkte des Arbeitsprogramms wird zudem gewährleistet, dass die Schwerpunkte zielgerichtet und strukturiert verwirklicht werden.

\subsection{Ausblick und Vorteile}

\section{Ergebnissicherheit und Optimierung der Betriebsabläufe}

Durch die prozess- bzw. systemorientierte Betrachtungsweise bei der Einführung eines QM-Systems werden die Betriebsabläufe eingehend analysiert und ihr Vorgehen durch die Darstellung der In-/Outputs, der Aktivitäten, der Schnittstellen und der benötigten Ressourcen optimiert und verdeutlicht. Aus der Betrachtung und Umsetzung dieser Betriebsabläufe in Verfahrensanweisungen bzw. SOPs resultiert eine sehr gute Verfahrensklarheit und Reproduzierbarkeit für alle Beteiligten. Auf diese Weise kann u. a. die Ergebnisqualität und Lebensdauer der Prüfmittel durch die »Lenkung der Prüfmittel« verbessert werden. So wird z. B. mit dem Aufnehmen der Spezifikationen und dem Schaffen/Einhalten des Kalibrierungs-/Wartungsprogramms der Prüfmittel gewährleistet, dass ihr Einsatz und ihre Tauglichkeit permanent sichergestellt sind. Ein Prüfmittel, welches stets mit einer systematischen Abweichung misst und nie überwacht wird, würde kontinuierlich falsche Messergebnisse lie- 
fern. Auf der Basis von falschen Ergebnissen würden so eventuell falsche wissenschaftliche Schlussfolgerungen getroffen werden. So wird durch die Prüfmittellenkung die Voraussetzung gegeben, bestmögliche Ergebnisse nahe dem realen Messwert zu erhalten.

\section{Schonung staatlicher Ressourcen und verbesserte Lage zur Akquirierung von Forschungs-/Kundenaufträgen}

Ein Beispiel zur Schonung der Ressourcen des Staates ist die konsequente Einhaltung der Prüfmittellenkung. Durch die Befolgung des Wartungsprogramms aus der Prüfmittellenkung werden die Prüfmittel entsprechend der Vorgaben gepflegt. Die Prüfmittel werden so langlebiger, was zu einer Schonung der bereitgestellten Ressourcen führt. Ein weiteres Beispiel zur Schonung staatlicher Ressourcen ist $u$. a. die getroffene Regelung zur Beschaffung von Ausrüstung und Material. Durch das Schaffen klarer Verfahrensabläufe und der Erfüllung der Anforderungen der Norm werden Doppelarbeiten vermieden, es herrscht Klarheit über die Bestände des Labors (z. B. Chemikalien und Geräte) und es werden entsprechend der geforderten Qualität nur geeignete Materialien beschafft.

Wenn der Staat/Kooperationspartner bei der Vergabe von Forschungsgeldern davon ausgehen kann, dass das Labor in einem hohem Maß vertrauensvoll mit den ihm übergebenen Ressourcen umgeht, wird das Labor möglicherweise bei der Vergabe bevorzugt behandelt werden. Zudem zeigt die kontinuierlich steigende Zahl der Akkreditierungen, dass Kunden zusehends ein höheres Vertrauen an die erbrachten Ergebnisse fordern.

\section{Praktischer Nutzen für die studentische Ausbildung} Mit der Einführung eines QM-Systems kann zudem die praktische Ausbildung der Studenten verbessert werden. Erkenntnisse aus der Einführung und Umsetzung können in Lehrveranstaltungen einfließen. Fortgeschrittene Studenten können bei einem Praktikum im Labor die Arbeit in einem QM-System erleben. Sie führen ihre Tätigkeiten entsprechend den Vorgaben des QM-Systems durch und dokumentieren diese dementsprechend. So wird es für sie möglich, industrienahe Erfahrungen beim Arbeiten in einem QM-System zu sammeln. Der Meinung der Industrie nach zu urteilen, fehlen Studenten häufig diese Erkenntnisse (Giese 2009).

\subsection{Fazit}

Die Auswahl des QM-Regelwerks ISO 9001 oder ISO 17025 muss individuell von dem Labor selbst getroffen werden. Mit der Einführung eines QM-Systems und der folgenden Betrachtung der einzelnen Prozesse unter Berücksichtigung der Anforderung aus der Norm entsteht eine verbesserte Verfahrensklarheit im Labor. Auf diese Weise werden die Arbeitsabläufe sowie die Ergebnisqualität positiv verbessert und staatliche Ressourcen geschont. Mit der Bestätigung durch eine Zertifizierung oder Akkreditierung wird zudem das Vertrauen in die Leistungsfähigkeit des Labors gesteigert, was die Aussichten für weitere Forschungs/-Kundenaufträge verbessert. Zudem können Studenten durch die gewonnenen Erfahrungen in einem QM-System ihre Chancen auf dem Arbeitsmarkt erhöhen. Die Einführung eines QM-Systems ist daher als eine Investition in die $\mathrm{Zu}$ kunft zu sehen, um den Erfolg für das Labor deutlich aussichtsreicher zu gestalten.

Übersteigen die jährlichen Kosten für eine Zertifizierung oder Akkreditierung das Budget, ist es nicht notwendig, sich einer externen Evaluierung zu unterziehen. Ein QM-System sollte aber dennoch eingeführt werden. Bei diesem Vorgehen würde die Norm dem eigenen Nutzen entsprechend umgesetzt werden. Dabei sollte die Norm als Leitfaden zur »Guten Praxis im Labor« dienen. Vorteile, die sich z. B. aus der Prüfmittellenkung oder Beschaffung ergeben, sollten umgesetzt werden. Weitere geforderte Prozesse aus der Norm, wie u. a. die »Prüfung von Verträgen « oder »Interne Audits«, könnten zunächst ausgelassen werden.

\section{Anmerkung}

Im Rahmen der erstmaligen Zertifizierung des QMSystems der Hochschule nach ISO 9001 wurde das QMSystem des Labors für Molekularbiologie und Funktionelle Genomik in Bezug auf die Prüfmittellenkung in die Auditierung der Hochschule einbezogen. Die Hochschule wurde im Juni durch die »Deutsche Gesellschaft zur Zertifizierung von Managementsystemen« (DQS) erfolgreich zertifiziert. Der Aufbau eines spezifischen QM-Systems für ein Labor hat somit Pilotcharakter für weitere Labore der Hochschule. 


\section{Literatur}

Brunner, F. J. und Wagner, K.W. (2008): Qualitätsmanagement: Leitfaden für Studium und Praxis, 4. überarbeitete Auflage, Kapitel 6/65, Carl Hanser Verlag München/Wien.

Cassel, M. (2000): Qualitätsmanagement nach ISO 9001:2000, Kapitel 6/1, Carl Hanser Verlag München/Wien.

DACH-N (2008): Nachrichten der DACH Nr. 1 2008, Webseite der DACH www.dach-gmbh.de/download/news/NACHR25.pdf.

DIN EN ISO 9000 (2005): Qualitätsmanagementsysteme. Grundlagen und Begriffe, www.beuth.de.

DIN EN ISO/IEC 17025 (2005): Allgemeine Anforderungen an die Kompetenz von Prüf- und Kalibrierlaboratorien, www.beuth.de.

Giese, C. (2009): Interview mit dem Abteilungsleiter Zell-, Gewebeservice und Qualitätskontrolle der Probiogen AG C. Giese. [Probiogen AG ist ein Biotechnologie Unternehmen mit GMP \& ISO 9001 Zertifikat; www.probiogen.de].

GLP-INFO (2004): Die GLP-Bundesstelle informiert - GLP-INFO 11, 11.08.2004; www.bfr.bund.de/cd/480:1-8.

Kamiske, G. F. und Brauer, J. P. (2008): Qualitätsmanagement von A bis Z: Erläuterungen moderner Begriffe des Qualitätsmanagements, 6. Auflage, Kapitel 2/218, Carl Hanser Verlag München.

QMV (2008): Musterdokument einer QM-Verfahrensanweisung zur "Messmittelüberwachung « der TFH-Wildau-Projektgruppe Qualitätsmanagement.

Malow, A. (2009): Interview mit einer Mitarbeiterin der Deutschen Gesellschaft zur Zertifizierung von Managementsystemen; 20.08.2009.

Sagert, M. (2003): Untersuchung zur Einführung eines Qualitätsmanagementsystems nach ISO 9000:2000ff in kleinen Unternehmen. 20.03.2003, Diplomarbeit an der TFH Wildau im Studiengang Wirtschaftsingenieurwesen; Reg.-Nr.W18/96(00).

Schweitzer, R. (2009): Seminar zum Qualitätsmanagement in der Life Science; Top 50 Seminarreihe der BioTop (15.01.2009) in der Technologiestiftung Berlin Ludwig-Erhard-Haus.

Wisser, S. (2009): Interview mit einem Mitarbeiter der DACH der Abteilung Prüflaboratorien, Inspektionsstellen und Produktzertifizierungsstellen, 20.06.2009.

Wohlfahrt, I. (2008): Qualitätsmanagement: Ausgewählte Abbildungen zum Studienfach« Vorlesungsscript Bachelor-Studiengang Luftfahrttechnik/Luftfahrtlogistik an der TFH Wildau.

[Diese Publikation beruht auf der Masterarbeit des Erstautors im Studiengang Biosystemtechnik/Bioinformatik und wurde im Labor für Molekularbiologie und Funktionelle Genomik durchgeführt.]
Autoren

M. Sc. Stefan Weinert

TH Wildau [FH]

Molekularbiologie und Funktionelle Genomik

stefan.weinert@tfh-wildau.de

Dr.-Ing. Ingolf Wohlfahrt

TH Wildau [FH]

Fertigungsmesstechnik und Qualitätsmanagement

ingolf.wohlfahrt@tfh-wildau.de

Dipl.-Psychologin Andrea Schmid

TH Wildau [FH]

Qualitätsmanagementbeauftragte

andrea.schmid@tfh-wildau.de

Prof. Dr. sc. hum. Marcus Frohme (korrespondierender Autor) TH Wildau [FH]

Molekularbiologie und Funktionelle Genomik

Tel. +493375 508-249

marcus.frohme@tfh-wildau.de 